\title{
MVRC Heuristic for Solving the Multi-Choice Multi-Constraint Knapsack Problem
}

\author{
Maria Chantzara and Miltiades Anagnostou \\ School of Electrical \& Computer Engineering, National Technical University of Athens \\ 9 Heroon Polytechniou Str, 15773 Zografou, Athens, Greece \\ \{marhantz, miltos\} atelecom.ntua.gr
}

\begin{abstract}
This paper presents the heuristic algorithm Maximizing Value per Resources Consumption (MVRC) that solves the Multi-Choice MultiConstraint Knapsack Problem, a variant of the known NP-hard optimization problem called Knapsack problem. Starting with an initial solution, the MVRC performs iterative improvements through exchanging the already picked items in order to conclude to the optimal solution. Following a three step procedure, it tries to pick the items with the maximum Value per Aggregate Resources Consumption. The proposed algorithm has been evaluated in terms of the quality of the final solution and its run-time performance.
\end{abstract}

\section{Introduction}

One of the most studied combinatorial optimization problems is the Knapsack Problem (KP). Numerous problems of different fields such as capital budgeting, cargo loading and resource allocation are modeled as a variant of it. Due to the high applicability of this NP-hard problem, it has been widely studied. The objective of the original KP is to optimize resource allocation, or more precisely, how to distribute a fixed amount of resources among several actions in order to obtain maximum payoff. The 0-1 KP considers a knapsack of specific capacity and a set of items; each of them has specific weight and value. The objective is to determine which items should be placed in the knapsack so as to maximize the total value of the items contained in it without exceeding its capacity. Another variant of the 0-1 KP refers to the case that there are multiple constraints regarding resources and is called Multi-Dimension or Multi-Constraint KP (MDKP). Another one is the Multi-Choice KP (MCKP). In this case the items are divided into groups and the objective is to pick exactly one item of every group in order to maximize the total value.

A combination of the MDKP and MCKP variants is the Multi-Choice MultiConstraint Knapsack Problem (MMKP). Its formal definition is: There are $\mathrm{n}$ groups of items. Group i contains $l_{i}$ items. Each item ij has a particular value $v_{i j}$ and weights $r_{i j}=\left(r_{1 j}, \ldots, r_{m i j}\right)$ regarding the $\mathrm{m}$ resource constraints: $C=\left(C_{1, \cdots, C_{m}}\right)$. The objective is to pick exactly one item from each group in order to have maximum total value of the collected items, subject to the $\mathrm{m}$ resource constraints. With decision variable: $x_{i j} \in\{0,1\}$, the MMKP is formulated as follows. The objective function to be maximized is the total value of the picked items: 


$$
\text { TotalValue }=\sum_{i=1}^{i=n} \sum_{j=1}^{j=l_{i}} v_{i j} x_{i j}
$$

The constraints are:

$$
\begin{gathered}
\sum_{i=1}^{i=n} \sum_{j=1}^{j=l_{i}} r_{k i j} x_{i j} \leq C_{k}, k=1, \ldots, m \\
\sum_{j=1}^{j=l_{i}} x_{i j}=1, i=1, \ldots, n
\end{gathered}
$$

This paper presents the heuristic Maximizing Value per Resources Consumption (MVRC) for solving the MMKP. It is an improvement of the heuristic HEU [1] which is proved to be one of the best known algorithms in terms of performance (solution optimality vs. computation time). Starting with an initial solution, MVRC performs iterative improvements though exchanging the already picked items in order to conclude to the optimal solution. Unlike HEU that tries to minimize the resources consumption, MVRC picks the items with the maximum Value per Aggregate Resources Consumption. Finally, MVRC solves the MMKP instances in less time that is important for cases that require real-time decision making. We have applied the proposed heuristic to the MMKP instance referring to the discovery of the information sources for acquiring data on behalf of the context-aware services. Regarding this problem, each requested type of info corresponds to a group, and the available quality levels of the same context data correspond to the items of the group. The resource constraints refer to the cost and the latency bound for obtaining the data. The value that needs to be maximized is the expected utility of the picked items. The formulation of context source discovery as MMKP has been analyzed in [2]. In this case, the values do not follow monotone feasibility order, since items with higher cost correspond to higher expected utility, while items with higher response time to lower.

The rest of the paper is organized as follows: the literature review related to MMKP is reported in Section 2. In Section 3, the proposed heuristic MVRC is detailed and its worst-case complexity is computed. In Section 4 the evaluation of the MVRC performance is presented. Finally, Section 5 provides some conclusive remarks.

\section{Related Work}

In [3] and recently in [4], reviews of the KPs and literature on methods to solve them are presented. The proposed exact algorithms for solving the different variants are based on the "branch-and-bound" approach or dynamic programming techniques, that are capable of producing optimal solutions but they require much time. Therefore, heuristics providing near-optimal solutions within low computation time are developed. We are particularly interested in the literature about the MMKP variant. The exact algorithm for the MMKP is a branch-and-bound [5] that performs a complete enumeration keeping the best solution found so far in order to find the optimal one. If a partial solution cannot improve on the best, it is abandoned. 
However, this approach cannot be applied when the selection should be done real-time, due to its high complexity and therefore, heuristics are developed.

One of the first heuristics for solving the MMKP is presented in [6]. It uses the concept of graceful degradation from the most valuable items based on Lagrange Multipliers. This algorithm fails to find a solution when the resources are extremely short and the feasible solutions are very few. In [1] the heuristic HEU is detailed. This approach is based on the concept of "aggregate resources" proposed in [7] that converts the multiple resource dimensions into only one through penalizing the use of resources. In fact, it applies a large penalty for a heavily used resource and a small penalty for a lightly used resource. The proposed method starts from an initial pick of the least valuable items, finds a feasible solution by exchanging items based on the Toyoda concept, while it ensures that there is improvement regarding the resources consumption. It finally upgrades the feasible solution in terms of solution value through iterative exchanges resulting to another feasible solution. The HEU has been applied to the QoS management problem, where the QoS levels follow monotone feasibility order since a QoS level with higher utility requires more resources. In order to have better solutions for the MMKP instances where some higher-valued items consume less resources than lower-valued, the authors of [8] have proposed another method. This one applies a transformation technique to map the multi-dimensional resource to single dimension and constructs convex hulls to reduce the search space of solutions. Comparing it with the HEU showed that even though it produces solutions with significant lower value especially for the correlated data sets, it concludes to the solution in significant reduced time. As a result, it can be stated that it mostly fits to cases where run-time performance is of greater interest than the solution quality. Finally, the authors of [9] propose a set of algorithms for finding the solution of an MMKP instance. The first one constructs an initial feasible solution through a greedy approach, the second one improves the quality of the initial solution using a complementary procedure and the third one searches for the best feasible solution over a set of neighborhoods according to the "guided local search" method. The evaluation of the performance of this approach showed that it outperforms the HEU in terms of the solution quality but for the run-time performance no metrics are given.

\section{The MVRC Heuristic Algorithm}

Before describing the MVRC heuristic, we introduce some notations. Considering item $\mathrm{ij}$ with value $\mathrm{v}_{\mathrm{ij}}$ and resources usage $r_{i j}=\left(r_{1 i j}, \ldots, r_{m i j}\right)$, we define the Aggregate Resources Consumption (ARC): $A R C_{i j}=\frac{r_{1 i j} * C_{1}+\ldots+r_{k i j} * C_{k}}{\sqrt{C_{1}^{2}+\ldots+C_{k}^{2}}}$, and the Value-per unit of ARC (V-ARC): $V-A R C_{i j}=v_{i j} / A R C_{i j}$. Assuming that we have the problem's solution described by the vector $S=\left(1 \mathrm{j}_{1}, 2 \mathrm{j}_{2}, \ldots, \mathrm{ij}_{\mathrm{i}}, . ., n \mathrm{j}_{\mathrm{n}}\right)$, denoting the items picked per each group, and resources consumption $\mathrm{R}_{\mathrm{S}}=\left(\mathrm{R}_{1}, \mathrm{R}_{2}, \ldots \mathrm{R}_{\mathrm{k}}\right)$ where $R_{k}=\sum_{i=1}^{i=n} r_{k i j_{i}}$. If the already picked item $\mathrm{ij}_{\mathrm{i}}$ of group $\mathrm{i}$ is exchanged by the item $\mathrm{ij}$, the new solution is: $S^{\prime}=\left(1 j_{1}, 2 j_{2}, \ldots, i j, \ldots, n j_{n}\right)$. The resources consumption becomes $R_{S^{\prime}}=\left(R_{1}^{\prime}, R_{2}^{\prime}, \ldots, R_{k}^{\prime}\right)$. 
For the new solution $\mathrm{S}^{\prime}$, we define the Aggregate Resources Requirements (ARR): $A R R_{i j}=\frac{\left(R_{1}^{\prime}-R_{1}\right) * R_{1}+\ldots+\left(R_{k}^{\prime}-R_{k}\right) * R_{k}}{\sqrt{R_{1}^{2}+\ldots+R_{k}^{2}}}$, and the Value Update-per unit of $A R R$ (VU-ARR): $V U-A R R_{i j}=\frac{v_{i j}-v_{i j}}{A R R_{i j}}$. For the solution $\mathrm{S}$, we also define the feasibility factor of each resource $\mathrm{k}$ as follows: $F_{k}=R_{k} / C_{k}$. If $F_{k} \leq 1$, the resource $\mathrm{k}$ is called feasible; otherwise it is infeasible. In the same respect, if $F_{k} \leq 1$ for each $\mathrm{k}=1,2, \ldots, \mathrm{m}$, the solution $\mathrm{S}$ is called feasible; otherwise it is called infeasible. Moreover for the picked items in solution $\mathrm{S}$, we define the feasibility factor of each item $\mathrm{ij}_{\mathrm{i}}$ of group $\mathrm{i}$ for each resource k, as follows: $F I_{k j_{i}}=r_{k i j_{i}} / R_{k}$. Finally, the sum of the values of the picked items is the solution value: $V_{S}=\sum_{i=1}^{i=n} v_{i j_{i}}$.

The MVRC heuristic follows three steps in order to reach the final solution. Given the MMKP input instance, Step1 produces an initial solution. Step 2 produces a feasible solution through iteratively exchanging the already picked items. In Step 3 the feasible solution is improved by iteratively picking items with higher values.

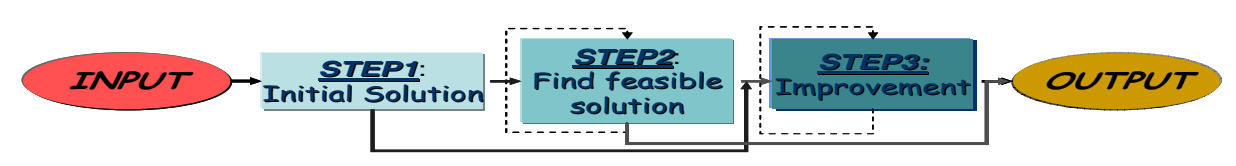

Fig. 1. MVRC heuristic

The MVRC steps are described in more detail below:

STEP 1: We find the initial solution by selecting the item with the highest V-ARC of each group. In case the solution is a feasible Step 3 follows; otherwise Step 2 follows. STEP 2: In this step, we exchange one of the items of the current solution in order to find a feasible solution. The decision about the exchange is the outcome of the following sub-steps. Firstly, the resource with the highest feasibility factor is found (step 2.1) and in relation to this resource, the picked item with the highest feasibility factor is determined (step 2.2). Then, we exchange this item with an item of the same group that has lower V-ARC (step 2.3). In case the solution which has come out of this exchange remains infeasible, Step 2 is repeated; otherwise, Step 3 follows. If Step 2 fails to find any feasible solution, the algorithm terminates without a solution.

STEP 3: In this step, we exchange one of the items of the current solution with another of the same group in order to increase the solution value. The criterion is the maximization of the VU-ARR ratio so that the new solution remains feasible. For deciding on the item to insert in the knapsack, we identify the following cases. Having two candidate items, with aggregate resources requirements $\mathrm{ARR}_{1}$ and $\mathrm{ARR}_{2}$ respectively: (i) If both $\mathrm{ARR}_{1}, \mathrm{ARR}_{2} \leq 0$, the item that maximizes the solution upgrade is preferred. (ii) If both $\mathrm{ARR}_{1}, \mathrm{ARR}_{2}>0$, the one with the maximum VU- 
$A R R$ is preferred. (iii) If $A R R_{1} \leq 0$ and $A R R_{2}>0$, the one that maximizes solution value upgrade is preferred. In case, a new exchange can be performed, Step 3 is repeated; otherwise, the algorithm terminates and the current solution is the optimal.

In the following, we present the upper bounds of the computational complexity of the three steps of the MVRC heuristic for the MMKP input instance consisting of $n$ groups, $\mathrm{m}$ resources and $\mathrm{l}$ items per group. It is assumed that in each group the items are arranged in non-decreasing order. In Step 1, for every item the ratio V-ARC is computed with complexity $\mathrm{O}(\mathrm{nml})$. For the sorting of the items according to the $\mathrm{V}$ ARC ratios we use a simple algorithm with complexity O(nl!). For the feasibility check the complexity is $\mathrm{O}(\mathrm{m})$. Therefore, the complexity of Step 1 is $\mathrm{O}(\mathrm{nml}+\mathrm{nl} !+\mathrm{m})$. The step 2.1 requires $m$ comparisons and is $O(m)$. The step 2.2 is $O(n)$. The step 2.3 requires constant time since the items are already ordered. The Step 2 can be repeated at most $\mathrm{n}(1-1)$. Thus, the complexity of Step 2 is $\mathrm{O}\left(\mathrm{n}^{2}(1-1) \mathrm{m}\right)$. In Step 3, computing the VU-ARR has $\mathrm{O}(\mathrm{m})$ complexity, while finding the item to be exchanged may require $\mathrm{n}(1-1)$ computations. Thus, the complexity is $\mathrm{O}(\mathrm{n}(\mathrm{l}-1) \mathrm{m})$. Since the feasible upgrades could be $\mathrm{n}(1-1)$ at most, the complexity of Step 3 is $\mathrm{O}\left(\mathrm{n}^{2}(1-1)^{2} \mathrm{~m}\right)$. Finally, we conclude that the complexity of the MVRC heuristic is $\mathrm{O}\left(\mathrm{nml}+\mathrm{nl} !+\mathrm{m}+\mathrm{n}^{2}(1-1) \mathrm{m}+\mathrm{n}^{2}(1-1)^{2} \mathrm{~m}\right)$.

\section{Evaluation}

In order to test the performance of the proposed algorithm, we implemented the MVRC algorithm along with the HEU and the exhaustive approach. The HEU is one of the best known heuristics that has been evaluated comparatively to all proposed approaches of the literature. The exhaustive approach is an exact algorithm that computes all possible combinations and checks their feasibility in order to find the optimal one (its complexity is $O(m l n)$ ). We applied the three algorithms to instances of different sizes under the scope to test both the quality of the solution and the runtime performance. The implementation of the algorithms is done in Java 1.4., while we ran the algorithms on a $1.4 \mathrm{GHz}$ Pentium Fujitsu Siemens Lifebook with 512MB running Linux. The data sets were generated according to the state-of-the-art pattern described in [8] that generated both correlated and uncorrelated data sets. Concerning the correlated data instances, that are harder to solve [10], the value of an item depends on its weights, while concerning the uncorrelated, the value and the weights are independent. The metrics measuring the performance of the algorithms are:

1. Percentage (\%) optimality of the solution that measures the quality of the produced solution. Assuming that $\mathrm{V}_{\mathrm{opt}}$ is the optimal one and $\mathrm{V}_{\mathrm{i}}$ is the one we wish to compare, the percentage optimality is defined as follows: $\% O p t=V_{i} / V_{\text {opt }} * 100$

2. Computation time describing the required time to execute the algorithm.

3. Number of updates that are performed till the final solution is found, showing how quick the algorithm converges to the final solution. Unlike the computation time, this metric does not depend on the implementation of the algorithm.

We recorded these metrics for all algorithms with the increase in the number of groups, items per group, resource constraints. For each size of data set, we generated 
10 instances and applied the algorithms to all of them. However, we report the average of the performance metrics of the 10 test instances of the specific data set size. The graphs of figures 2-4 depict the \% optimality of the solutions for small-sized data sets, since we are unable to run the exhaustive approach for large-sized data instances due to memory and computation time requirements. The graphs of figures 5-7 depict the computation time for the two algorithms to find a solution for large-sized data instances. Finally, the figures 8-10 depict the number of updates respectively.

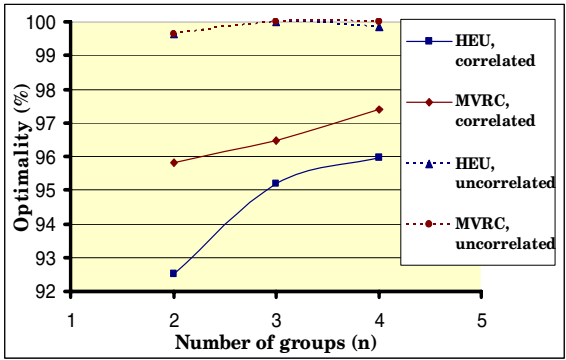

Fig. 2. Optimality in relation to the number of groups $(\mathrm{l}=20, \mathrm{~m}=2)$

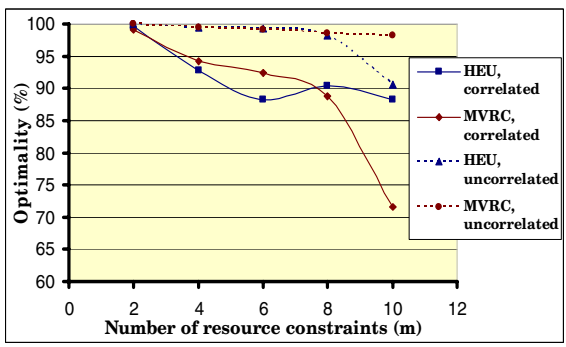

Fig. 4. Optimality in relation to the number of Fig. 5.Computation time in relation to the res. constraints $(n=2, l=20)$

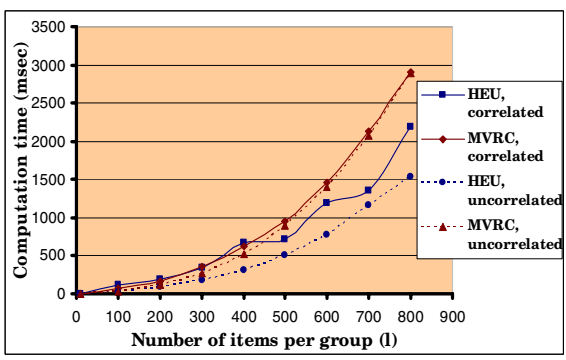

Fig. 6. Computation time in relation to the number of items per group $(n=10, m=2)$

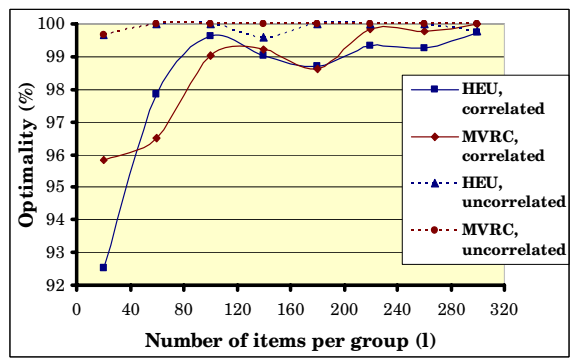

Fig. 3. Optimality in relation to the number of items per group $(n=2, m=2)$

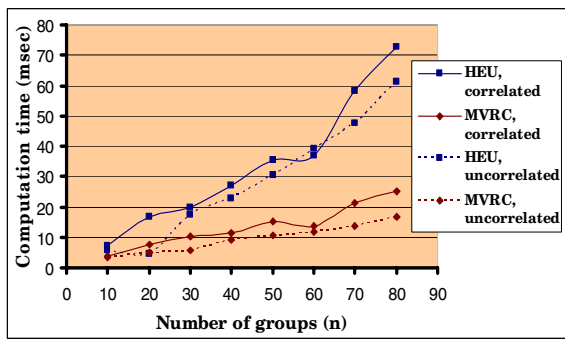
number of groups $(1=10, m=2)$

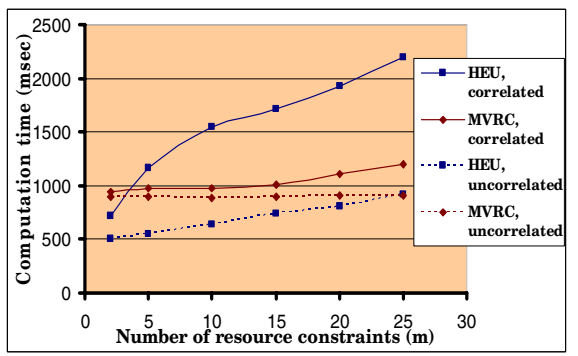

Fig. 7. Computation time in relation to the number of resource constraints $(n=10,1=500)$ 


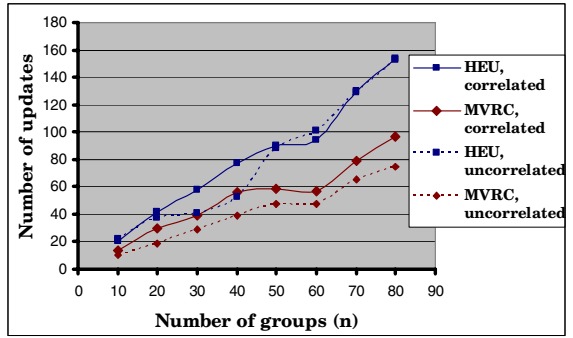

Fig. 8. Number of updates in relation to the number of groups $(\mathrm{l}=10, \mathrm{~m}=2)$

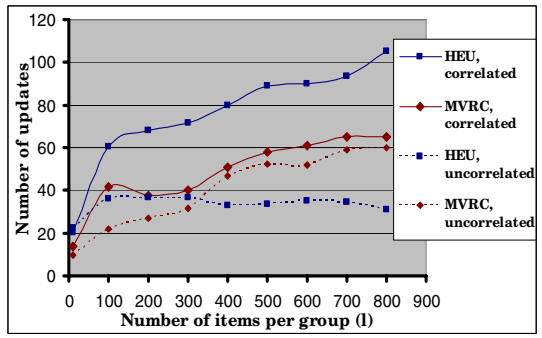

Fig. 9. Number of updates in relation to the number of items per group $(n=10, m=2)$

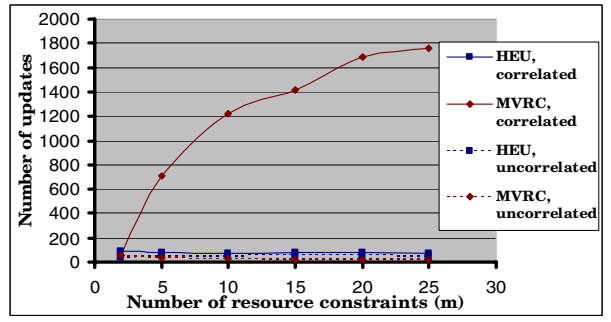

Fig. 10. Number of updates in relation to the number of resource constraints $(n=10,1=500)$

Observing the above figures we conclude to the following evaluation results:

- Testing the algorithms with small-sized data instances showed that both algorithms provide feasible solutions for every case. However, using large-sized input instances the algorithms showed that MVRC fails to find feasible solutions for large number of resource constraints. In fact, for $n=10,1=500, m=20$, the failure rate is $50 \%$ while for $\mathrm{m}=25$, it becomes $70 \%$. For the other large-sized input instances, no failure is noticed.

- Regarding the quality of the solutions, we conclude that both algorithms produce solutions with value very close to the one produced by the exhaustive approach. For the smaller data instances, the increase of the problem size result in the increase of optimality, while for larger data instances the optimality tends to stabilize. Regarding the quality of the solutions for large data sets, we observe that the solutions produced by the two algorithms are quite close.

- It may happen that smaller data sets take longer than larger data sets. This happens because of the fact that the data sets are produced randomly. If the data set consists of very few feasible solutions it might take less time to get the final solution.

- The performance for the uncorrelated data sets is better than the correlated ones.

- The MVRC outperforms the HEU in terms of solution optimality. It also performs less updates till finding the final solution, and requires lower computation time. As the data sizes increase, the increase rates of computation time and performed updates get higher for the HEU, while for the MVRC it tends to remain stable. The 
complexity of the HEU is quadratic to the group size and number of groups, while for the MVRC only the complexity of Step 3 is quadratic to the problem size. However, for large number of constraints the HEU produces better solutions in terms of quality but it requires more time.

- For large number of items per group, the computation time of the MVRC is higher than the HEU. However, this is due to the sorting algorithm with complexity $\mathrm{O}(\mathrm{nl}$ !) that is used in Step 1. A sorting algorithm with lower complexity, such as "Quicksort" [11], can be applied in order to minimize the computation time of the MVRC.

\section{Conclusions}

We presented the Maximizing Value per Resources Consumption heuristic algorithm for solving the MMKP within low time that is important for cases that require realtime decision making. The proposed algorithm has been tested against the HEU and the exhaustive approach for various sizes of input data. The evaluation results showed that the MVRC generates high quality solutions within low computation time. As the input data sizes increase, the increase rate of the computation time remains stable. However, it falls short in cases of large number of resource constraints. Moreover, in cases of large number of items per group it requires high computation time, but this can be improved if we utilize sorting algorithms with lower complexity.

\section{References}

1. Khan, S., Kin, F. Li, Manning, E., Akbar, M.: Solving the Knapsack Problem for Adaptive Multimedia Systems. Studia Informatica (2002), Special Issue on Combinatorial Problems. Vol. 2. No. 1, pp. 154-174.

2. Chantzara, M., Anagnostou, M.: Evaluation and Selection of Context Information. In Proceedings of the 2nd International Workshop on Modelling and Retrieval of Context (MRC 2005), Edinburgh, Scotland, (July 31- August 1 2005). CEUR Workshop Proceedings, ISSN 1613-0073.

3. Martello, S., Toth, P.: Knapsack Problems: Algorithms and Computer Implementations. Wiley-Inrescience Series in Discrete Mathematics and Optimization, 1990, John Wiley \& Sons, Chichester, England.

4. Kellerer, H., Pferschy, U., Pisinger, D.: Knapsack Problems. Springer, 2004 ,ISBN: 3540-40286-1, p. 546.

5. Khan, S.: Quality Adaptation in a Multi-Session Adaptive Multimedia System: Model, Algorithms and Architecture. PhD Thesis, Department of Electronical and Computer Engineering, University of Victoria, Canada (1998).

6. Moser, M., Jokanovic, D., Shiratori, N.: An Algorithm for the Multidimensional MultipleChoice Knapsack Problem. IECE Trans Fundamentals Electron (1997), Vol.80. pp. 582-589.

7. Toyoda, Y.: A Simplified Algorithm for Obtaining Approximate Solutions to Zero-one Programming Problems. Management Science (1975), Vol. 21, No. 12, pp. 1417-1427. 
8. Akbar, M., Rahman, M., Kaykobad, M., Manning, E., Shoja, G.: Solving the Multidimensional Multiple-choice Knapsack Problem by Constructing Convex Hulls. Computers \& Operations Research, Elsevier (May 2006). Vol. 33, No. 5, pp. 1259-1273.

9. Hifi, M., Micrafy, M., Sbihi, A.: Heuristic Algorithms for the Multiple-choice Multidimensional Knapsack Problem. Journal of the Operational Research Society (December 2004). Vol. 55, pp. 1323-1332.

10. Pisinger, D.: Where are the hard knapsack problems?. Computers and Operations Research (September 2005). Vol. 32, No. 9, pp. 2271-2284.

11. Cormen, T., Leiserson, C., Rivest, R., Stein, C.: Introduction to Algorithms. MIT Press and McGraw-Hill. 